\title{
Chapter 2 \\ Working with magnetic resonance images of the brain
}

\subsection{Human brain anatomy}

The human brain consists of multiple structures, including the large cerebrum, the smaller cerebellum, and the brain stem. These structures can easily be identified from MR images of the brain (Figure 2.1). The cerebrum is composed of the left and right hemispheres, which are connected through bundles of nerve fibers (the corpus callosum).

There are two main types of brain cells: neurons (or nerve cells) and glial cells. Each neuron is generally composed of a cell body (soma), a long nerve fiber (axon), and other branching extensions (dendrites). The spatial distribution of the brain cells results in two primary types of brain tissue matter: white and gray matter. ${ }^{1}$ White matter mainly consists of bundles of (myelinated) axons while gray matter includes neuronal cell bodies, and glial cells. The distribution of white and gray matter in the cerebrum is shown in Figure 2.1 (right), which demonstrates the white matter and the cortical and sub-cortical gray matter. The sub-cortical gray matter includes a number of

\footnotetext{
${ }^{1}$ In simple terms, neurons have a cell body (soma) and branches (axons and dendrites) that extend from the cell body. Axons are either covered with a lipid-rich (fatty) layer called myelin (myelinated axons) or surrounded by other cells (unmyelinated axons); myelin helps the axons conduct electrical signals over long distances. Myelin, being fatty, gives off a white-ish color; conversely, unmyelinated axons, dendrites, and neural cell bodies, in close proximity to microcirculation, appear gray. This is the origin of the terms gray matter and white matter.
} 

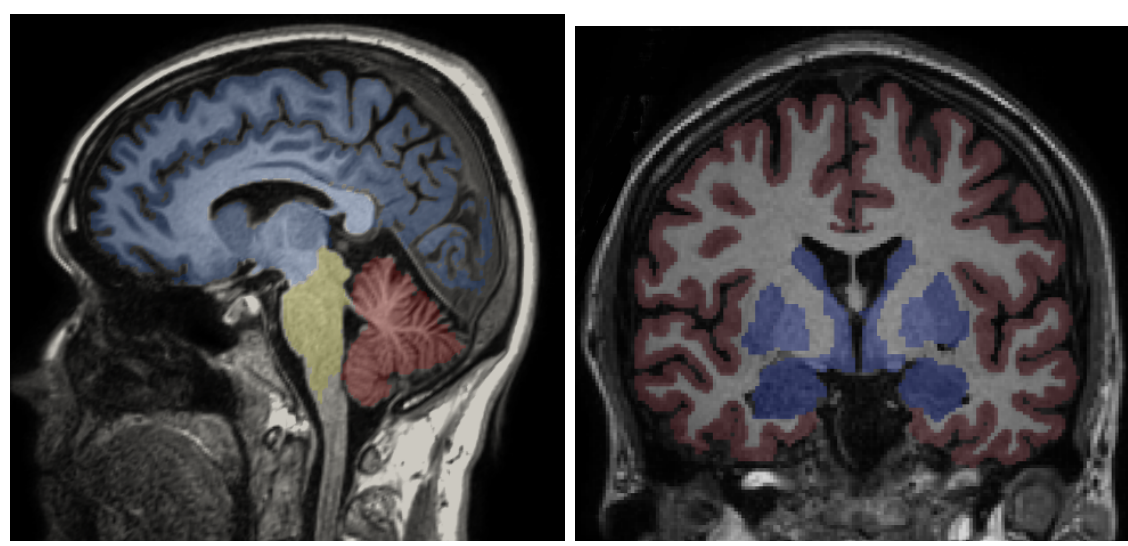

Fig. 2.1 MR images (T1-weighted) of the human brain. Left: A sagittal (longitudinal) cross-section of the human brain with the cerebrum in blue, the cerebellum in red, and the brain stem in yellow. Right: A coronal slice of the brain. The tissue matter composition of the cerebrum is marked by color with red denoting the cortical gray matter, blue denoting the subcortical gray matter and white denoting the white matter. (The colors were added after post-processing.)

important structures or regions located deep inside the brain, such as the hippocampus, basal ganglia and thalamus.

The brain is enclosed and protected by three layers of meninges; the outermost dura, the middle arachnoid and the innermost pia membrane. The narrow space between the pial and arachnoid membranes is filled with cerebrospinal fluid (CSF) and is referred to as the subarachnoid space (SAS). The SAS extends around the brain and further down along the spinal cord. The SAS is also connected to the ventricular system, a system of interconnected CSF compartments surrounded by the brain. The ventricular system consists of the two lateral ventricles and the third and fourth ventricles, shown in Figure 2.2. The thin passage between the third and fourth ventricle is known as the cerebral aqueduct.

We refer the reader, for example, to [26] for a good introduction to human brain anatomy and [13] for a general introduction to human physiology. 


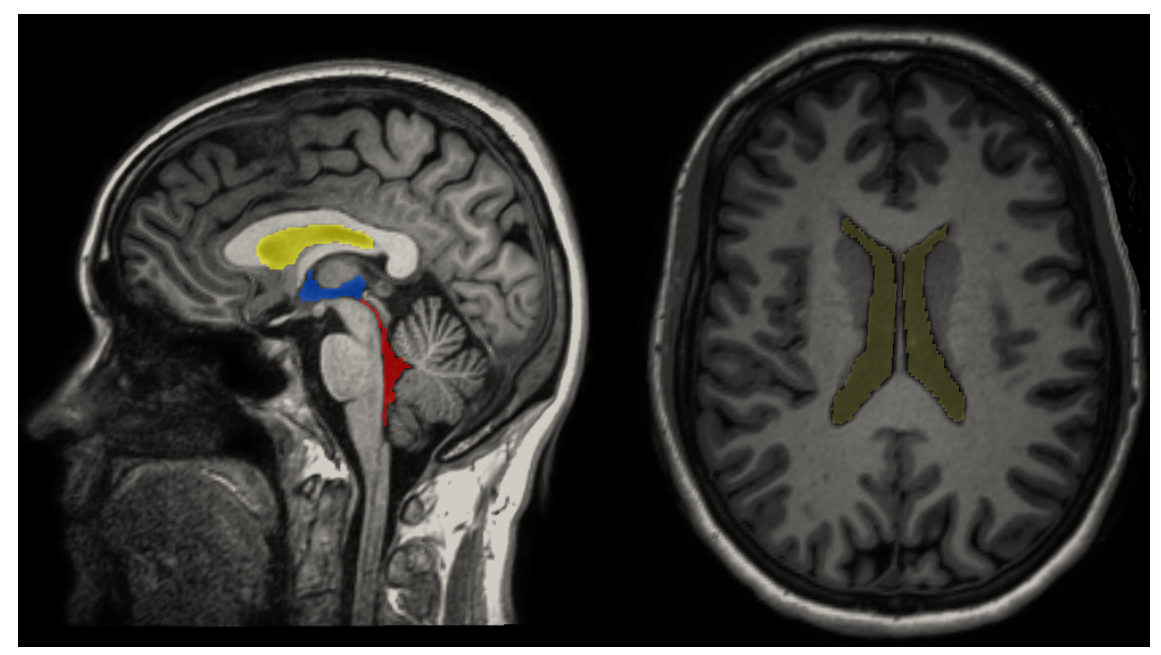

Fig. 2.2 Sagittal (left) and axial (right) MR image cross-sections of the brain with the lateral ventricles marked in yellow, the third ventricle marked in blue and the 4th ventricle and the aqueduct marked in red. The white region surrounding the lateral ventricles, on the left, is the corpus callosum. The distinction between (cortical) gray and white matter is also clearly visible on the right.

\subsection{Magnetic resonance imaging}

Magnetic resonance imaging (MRI) is a rich and versatile technique for the non-invasive medical imaging of the brain and other organs. The method has its roots in studies of Isidor Rabi; dating to the early 20th century. Rabi was able to ascertain information on the rotation and magnetic movements of the nuclei of atoms and molecules [65]. MRI leverages these types of magnetic properties; specifically for the nucleus of elemental hydrogen, which is abundant in fat and other tissues [12]. An MRI scanner creates a strong magnetic field, aligning the poles of hydrogen atoms along the scanner's axis. A radio wave is added to the magnetic field, causing the hydrogen nuclei to resonate, and different (scanner) slices of the body resonate differently. Turning off the applied radio frequency induces the realignment of the hydrogen nuclei with the applied magnetic field, and this process in turn causes the emission of another radio wave signal which the MRI scanner measures. The intensity of this last signal is what we visualize as MRI images [12]. 
The MRI method can be used for detailed investigations of tissue morphology and structure (structural MRI), tissue properties (e.g. diffusion-weighted MRI), blood flow (perfusion MRI) as well as aspects relating to brain function (functional MRI). As detailed above, an MR image is constructed from the interaction between a strong magnetic field and radio waves. The specifics of the procedure can be controlled by manipulating factors that affect this interaction, such as the magnetic field gradient. A specific set of changing magnetic gradients is referred to as an MRI sequence, which in turn has a number of parameters. We briefly describe a few key MRI sequences here; the interested reader can find more information in e.g. [29, 50, 8].

\subsubsection{Structural MRI: T1- and T2-weighted images}

Structural MRI provides high-resolution images of brain anatomy and can thus give information about the shape, size and composition of different brain compartments and regions ${ }^{2}$. Examples of structural MRI sequences include T1- and T2-weighted images, as already encountered in Figures 2.1 and 2.2. Such images are well suited for defining brain geometry models and will be used extensively for this purpose (Chapters 3-4). A brief introduction to T1and $\mathrm{T} 2$-weighted images can be found in [52].

T1- and T2-weighted images correspond to different (groups of) MRI sequences, each with their own parameters and characteristics. A T1- or T2weighted image is a three-dimensional image, typically viewed as a stack of black and white images of different planes (axial, coronal or sagittal) of the brain. The colored shading is referred to as the signal intensity; white represents a high signal intensity and black represents low intensity (with gray tones representing intermediate values). Both imaging sequences (T1- and T2weighted) produce different signal intensities for different types of tissue and fluids, but the two differ in their dominant intensities.

In T1-weighted images, fat gives off a high intensity signal and appears white, while fluids give off a low intensity signal and appear black. Therefore, the brain tissue appears as different shades of gray with white matter appearing lighter than gray matter (Figure 2.3 (left)). T1-weighted images are effective at differentiating between white and gray matter, but less effective

${ }^{2}$ Gray matter (cortical) and white matter regions are defined vis-a-vis a segmentation and parcellation process. Parcellations are discussed in more detail in Chapter 4.2.1 

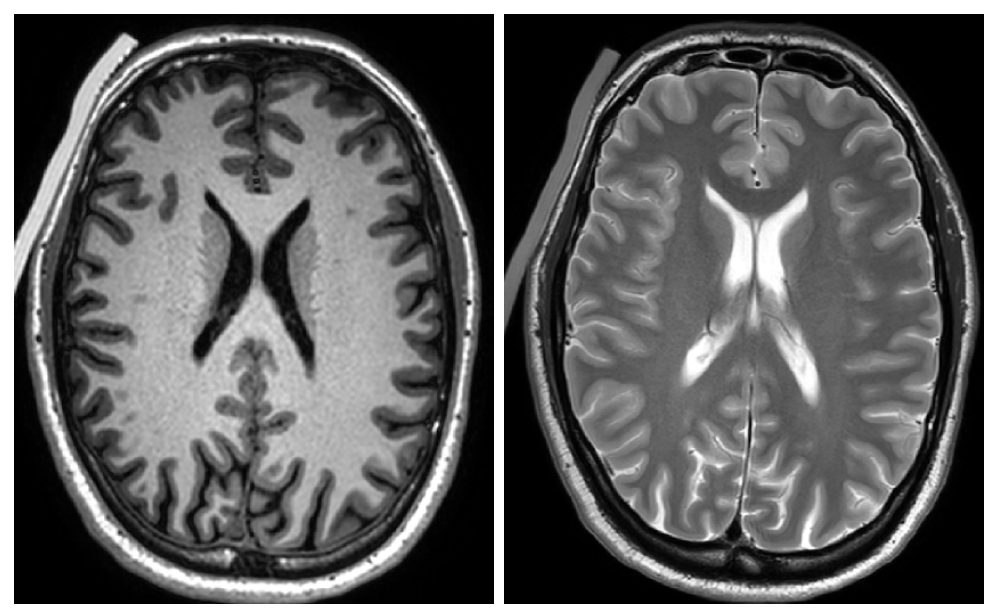

Fig. 2.3 T1-weighted image (left) versus T2-weighted image (right). In the T1weighted image, white matter is recognized as light gray, whereas the darker gray lining the surface of the brain is gray matter. T1-weighted images are used particularly because they exhibit a sharp contrast between gray and white matter. The T2-weighted image shows the CSF as almost white and provides good contrast between the CSF and the brain, but less contrast between white and gray matter. Also note that blood is dark in T2-weighted images.

at distinguishing between, for example, the CSF (black) and the gray matter (dark gray). In particular, it can be difficult to identify fluid compartments such as the ventricles, the aqueduct, and SAS from T1-weighted images alone.

In T2-weighted images on the other hand, fluids give off a high intensity signal and appear white (Figure 2.3 (right)). Such images are less effective at distinguishing between white and gray matter, but can provide good contrast between the CSF (white) and brain matter (gray). T2-weighted images can thus supplement data from T1-weighted images for identifying and separating the ventricles and aqueduct from subcortical gray matter.

\subsubsection{Diffusion-weighted imaging and diffusion tensor imaging}

Diffusion MRI (dMRI) is an imaging modality that detects water molecule movement patterns $[37,60]$. Both isotropic and anisotropic diffusion coeffi- 
Fig. 2.4 A raw, axiallyoriented dMRI image of the brain. The ventricles (center) appear in lower contrast due to the fast signal decay caused by the strongly isotropic diffusion of the water contained within them.

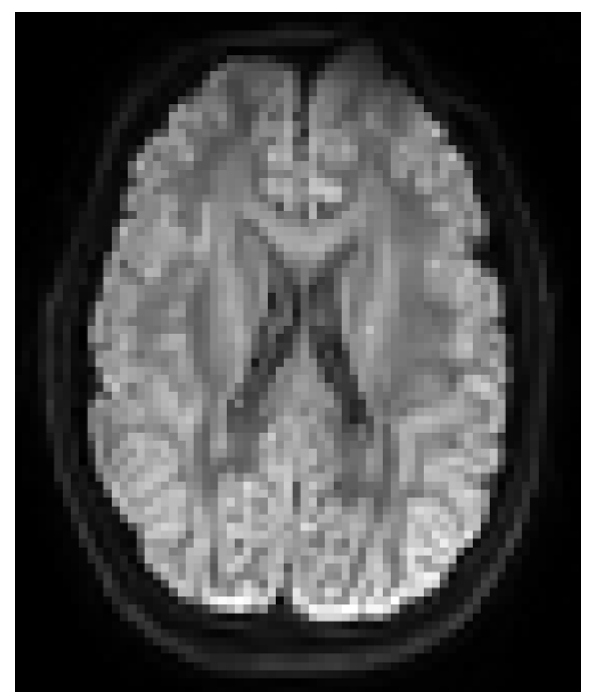

cients can be determined. Diffusion tensor imaging (DTI) is a specific type of diffusion-weighted MRI; Figure 2.4 shows an example of a DTI-weighted image. At a high level, a reference signal is used as a comparison and the DTI imaging process measures the difference in that reference signal with several follow-up signals. The resulting sequence provides information about how water diffuses in different regions of the brain.

More specifically, DTI provides information on both the magnitude and (multiple) directions of the movement of molecular water in the brain; that is, how water travels. In mathematical terms, DTI provides information regarding the diffusion tensor, $D$, in (1.1). In three dimensions, this tensor has nine entries and a global representation given by

$$
D=\left(\begin{array}{lll}
d_{11} & d_{12} & d_{13} \\
d_{21} & d_{22} & d_{23} \\
d_{31} & d_{32} & d_{33}
\end{array}\right) .
$$

Each of the entries $d_{i j}$ can be a function of the position $x \in \mathbb{R}^{3}$. We will extract the heterogeneous, anisotropic, and patient-specific diffusion tensor from DTI image data in Chapter 5. DTI has been used extensively to study the layout of brain's white matter tracts; these tracts heavily bias the movement of water within the brain. We refer the interested reader to [37], and the many sources 
therein, for a discussion of the more advanced topics related to the field of dMRI techniques.

\subsection{Viewing and working with MRI datasets}

\subsubsection{The DICOM file format}

Medical images, including T1, T2 and DTI, are often stored in the DICOM file format. DICOM stands for Digital Imaging and Communications in Medicine and is an imaging standard [48]. The format stores both the image itself and a comprehensive set of meta-data, such as the imaging protocol and patient identification, which enables consistent and safe usage across different vendors and software packages.

The DICOM format gives the output from an MRI scan as a collection of files arranged in sequences. A given file within a sequence ${ }^{3}$ also contains the necessary information to inform the viewer what the next (or previous) file in that file sequence is. DICOM files are sometimes stored in a binary file named DICOMDIR that indexes the entire structure of an MRI dataset.

\subsubsection{Working with the contents of an MRI dataset}

A DICOM viewer is an essential component for viewing and working with DICOM files. A number of DICOM browsers exist, and we will use DicomBrowser [11]. Currently, DicomBrowser is available ${ }^{4}$ as a pre-packaged binary download for several operating systems; the source code ${ }^{5}$ is also available. On

\footnotetext{
${ }^{3}$ Formally, the term MRI sequence does not refer to an ordered measure (such as a sequence in time) but rather to a specific type of pulse sequence or field gradient that determines the specific imaging protocol. A T1 MRI sequence produces T1 images, a T2 MRI sequence produces T2 images, and so forth. Here, when we speak of 'a sequence of files' we are referring to the order in which the files are produced by the MRI scanner; this is typically reflected in the naming of the files such that IM_0001 would be produced before IM_0002, and so on.

${ }^{4}$ https://wiki.xnat.org/xnat-tools/dicombrowser

${ }^{5}$ https://bitbucket.org/xnatdcm/dicombrowser/src/master/
} 
Ubuntu Linux, DicomBrowser comes pre-packaged as a Debian package file; this type of file can be installed on Ubuntu using the command

$$
\text { \$ sudo apt install /path to file/my-dicombrowser.deb (Ubuntu) }
$$

where you should change 'path to file' to the path where the DicomBrowser installation file (ending in .deb) has been downloaded and change the text of 'my-dicombrowser.deb' to be the specific filename of the DicomBrowser installer package.

You can then begin the process of extracting sequences from an MRI dataset by launching the DicomBrowser utility with the command:

\section{\$ DicomBrowser \&}

After the DicomBrowser window opens, select File $\rightarrow$ Open from the top menu bar; a dialogue box will appear with the heading Select DICOM files. Navigate to the directory containing the (example) book dataset and select the directory titled dicom/ernie. In this directory, select the file titled DICOMDIR. The main DicomBrowser window should now show a list of the patients whose data are included in this dataset (in this case just one patient). In the main window (c.f. Figure 2.5 (left)), click the patient, whose ID starts with 1.3.46, and then click the symbol to the left of the patient ID to expand the entry.

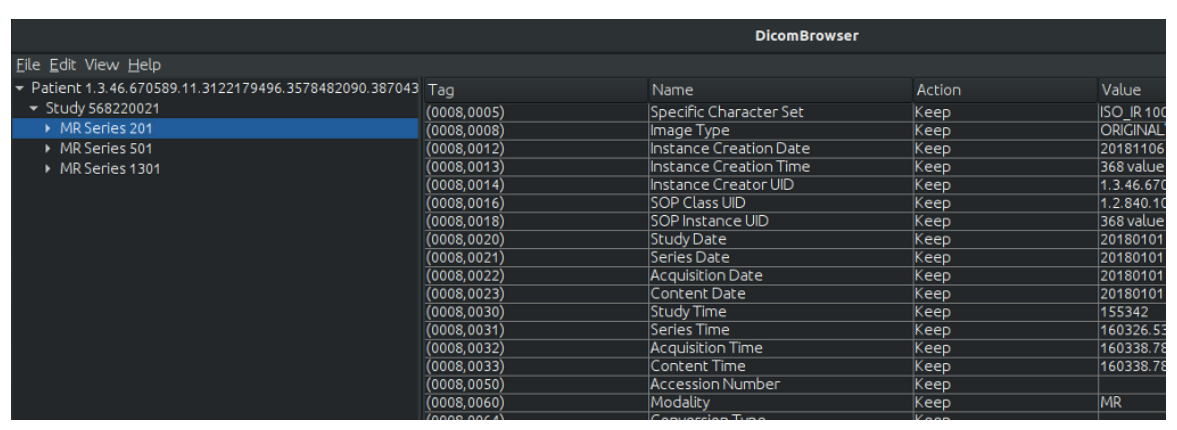

Fig. 2.5 Example of the DicomBrowser layout and selection of an MR series (dicom/ernie).

A list of studies now appears under the patient ID; generally, a patient can have several associated studies but the (example) book dataset contains 
one study per patient. Expand the study (beginning with 568) by clicking the symbol to the left. A list of MR series now fills the main window; many MR series can be contained in a single study and we see that the dicom/ernie data contains three series (201, 501 and 1301), as shown in Figure 2.5.

Find MR Series 201 and left-click on it. The secondary window now contains a list of tags, each of which has an associated name, action, and value (Figure 2.5). Note that tag $(0018,1030)^{6}$ is named 'protocol name' and has value T13D; this indicates that MR Series 201 corresponds to a T1-weighted image sequence. With 'MR Series 201' as the current selection, in the DicomBrowser (left) window, we can select File $\rightarrow$ Save to extract the series; a window then appears which provides extraction options. We would like to extract this series to a directory with a memorable name; we can do this by changing '-anon' to '-T13D' to signify that the series we are extracting represents a T1-weighted image in 3D. Also note that DicomBrowser can be used to anonymize the data, as indicated by the default extension -anon, or, in general, to change DICOM tag values either through the GUI or in scripts.

We can use the same procedure to extract other image sequences. In the DICOM images in our example dataset, MR Series 501 is a DTI sequence and MR Series 1301 includes T2-weighted images. We have already extracted all three series present in the sample DICOMDIR into the (example) book dataset. Image series 201 has been extracted to dicom/ernie/T13D, image series 1301 has been extracted to dicom/ernie/T23D, and image series 501 has been extracted to dicom/ernie/DTI. We note that dicom/ernie/DTI also contains some non-image files; these files are not in the DICOMDIR data but will be generated and discussed in Chapter 5.

\subsection{From images to simulation: A software ecosystem}

In this section, we provide a brief overview of the software tools that comprise the pipeline used in this book. We will extract data from clinical images, segment the data, extract and work with diffusion tensor information, generate a finite element mesh, and bring all of this together to solve a partial differential equation. Along the way, we will need additional tools for miscellaneous

\footnotetext{
6 Tag identifiers are not standard, and can differ based on the MRI scanner manufacturer. Generally, we identify a sequence from the Name field by looking for a label containing T1, T2, or DTI.
} 
objectives, such as file conversion and visualization. A visual representation of the patient-specific pipeline from MRI-images to finite element simulations is shown in Figure 2.6.

\subsubsection{FreeSurfer for MRI processing and segmentation}

FreeSurfer [18] is an open-source software suite for the segmentation (identification of different brain regions), processing, visualization, and analysis of human brain MR images. FreeSurfer is well established, well documented, and widely used and we refer to the FreeSurfer website for extensive documentation, online tutorials, support, an overview of publications and general installation instructions [2]. Generally, all FreeSurfer commands also have extensive documentation available via --help. At the time of writing, FreeSurfer provides step-by-step installation guides for Linux ${ }^{7}$ and Mac $^{8}$ Below, we discuss the Linux installation process, for the sake of completeness.

To install FreeSurfer on Ubuntu Linux, we suggest downloading the most recent FreeSurfer tar archive ${ }^{9}$ locally, for instance under /home/me/local/src. If the name of this file is freesurfer.tar.gz, unpack the archive via

$\$$ tar -zxvpf freesurfer.tar.gz

(Ubuntu)

The next step is to configure your environment for using FreeSurfer. If your FreeSurfer archive has been unpacked at /home/me/freesurfer, you can configure your FreeSurfer environment manually by adding the following lines at the end of the file named . bashrc in your home directory:

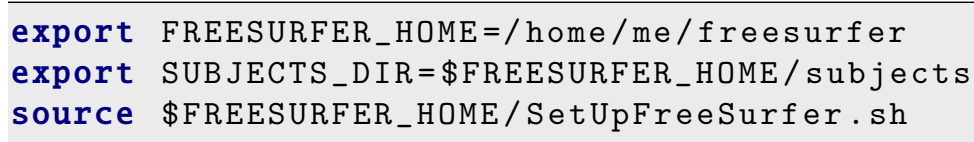

The FreeSurfer programming team requires that each user acquire a license file to use the software; the license file is free, and to acquire it, follow the registration directions at the FreeSurfer website [2]. Finally, FreeSurfer comes pack-

\footnotetext{
7 https://surfer.nmr.mgh.harvard.edu/fswiki//FS7_linux

8 https://surfer.nmr.mgh.harvard.edu/fswiki//FS7_mac

${ }^{9}$ See for example https://surfer.nmr.mgh.harvard.edu/fswiki/FS7_linux
} 


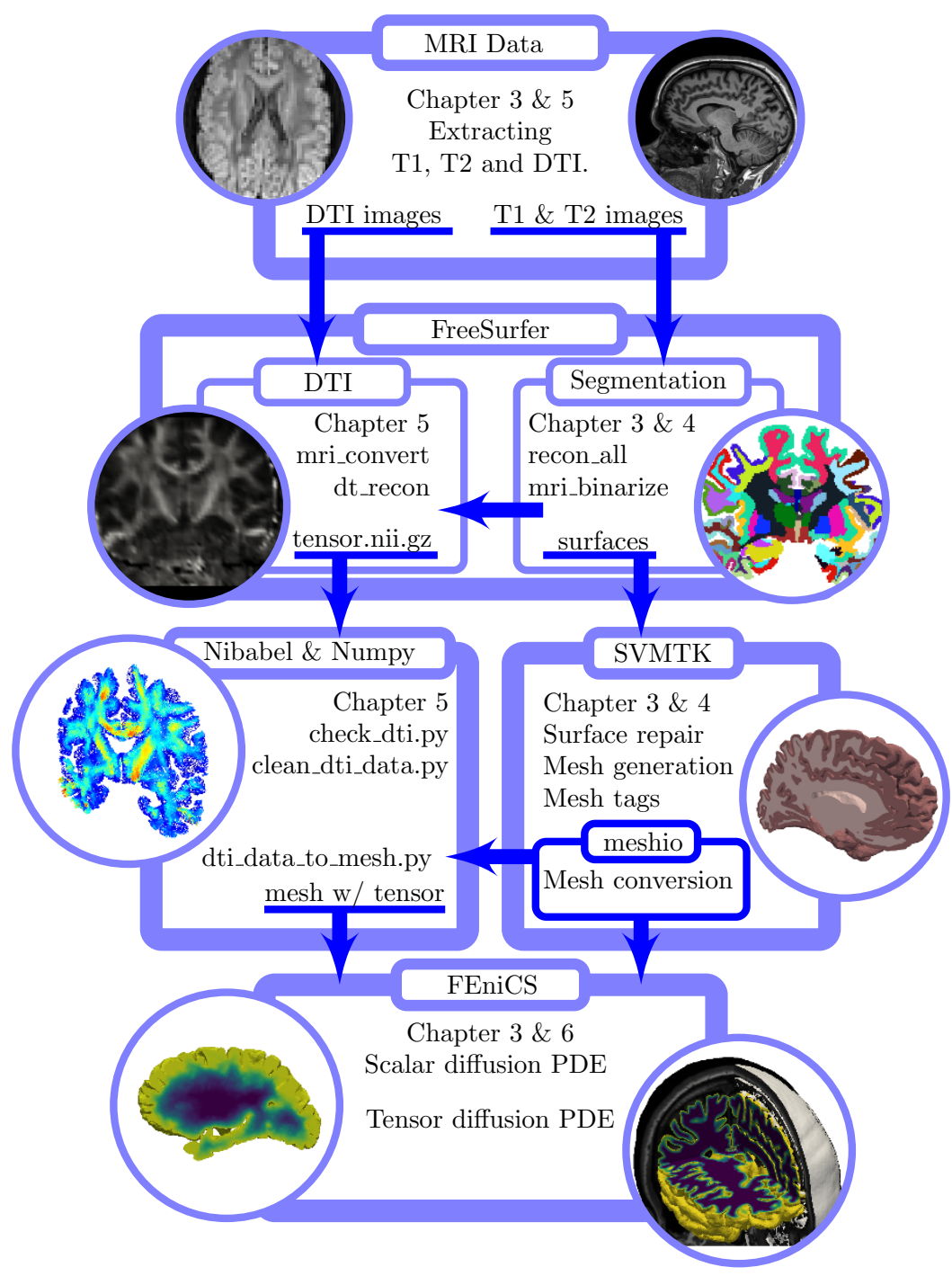

Fig. 2.6 Overview of the imaging, segmentation, meshing, simulation and visualization pipeline. T1, T2, and DTI images have already been discussed. Freesurfer and segmentation are discussed in Section 2.4.1. Nibabel and Numpy are Python libraries that are used for neuroimaging applications and convenient manipulation of tensors, respectively. SVM-Tk is a computational geometry package, written for this book, which is specialized for creating brain meshes and FEniCS is a Python library for high-performance finite element method computations. 
aged with the Freeview visualization tool. Test Freeview (and your FreeSurfer installation) by opening a new terminal (noting the FreeSurfer environment commands appearing on top), and typing:

\section{\$ freeview \&}

to open the Freeview user interface.

We will use the command dt_recon in FreeSurfer, which has additional requirements: $\operatorname{tcsh}^{10}$ and FSL. The installation of tcsh can be done from the terminal with the following command lines:

$\$$ sudo apt update

\$ sudo apt install tcsh

(Ubuntu)

FSL is a comprehensive library for functional MRI, MRI, and DTI brain imaging data [35], and we refer the reader to its website for installation instructions. ${ }^{11}$ Note that FSL, like FreeSurfer, requires a license to be operational.

\subsubsection{NiBabel: A python tool for MRI data}

The Python module NiBabel [14] provides read and write access to several neuroimaging file formats. The module is part of NIPY, ${ }^{12}$ a community for neuroimaging data analysis via Python. NiBabel can be installed using pip, for example, via the following terminal command:

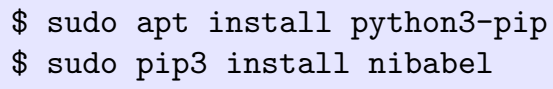

We will use Nibabel to work with image data in Python in Chapter 4 and onwards.

\footnotetext{
10 tcsh refers to a specific type of Unix shell. A Unix shell is a command-line interpreter; many such shells can be used in a Unix environment (such as Ubuntu Linux).

${ }^{11}$ See https://fsl.fmrib.ox.ac.uk/fsl/fslwiki/FslInstallation.

12 See https://nipy.org/.
} 


\subsubsection{SVM-Tk for volume mesh generation}

The Surface Volume Meshing Toolkit (SVM-Tk) generates meshes with subdomains based on surfaces and segmentations provided by FreeSurfer. In particular, SVM-Tk provides a Python-based interface to the Computational Geometry Algorithms Library (CGAL) [21] and provides tools for both fixing and marking surfaces to enable a relatively robust mesh generation for the various compartments of the brain. We refer to the SVM-Tk documentation ${ }^{13}$ for general installation instructions, including a detailed list of dependencies (including CGAL and a number of packages available, e.g. through the Ubuntu package manager).

\subsubsection{The FEniCS Project for finite element simulation}

We will use the open-source FEniCS Project $[9,44]$ as our finite element software. FEniCS includes both a $\mathrm{C}++$ and a Python interface; we will use the Python interface throughout. Extensive documentation, support, an overview of publications, and general installation instructions can be found on the FEniCS Project website [1]. In particular, we strongly encourage the reader to become familiar with FEniCS and the finite element method via the introductory FEniCS tutorial [41].

There are many ways to install the FEniCS Project software, including the use of Docker images, using pre-built Anaconda packages, or from source. Two simple ways of installing FEniCS are via Docker images (see the FEniCS Project website ${ }^{14}$ ) and via Ubuntu package managers. For the latter, use the following terminal commands:

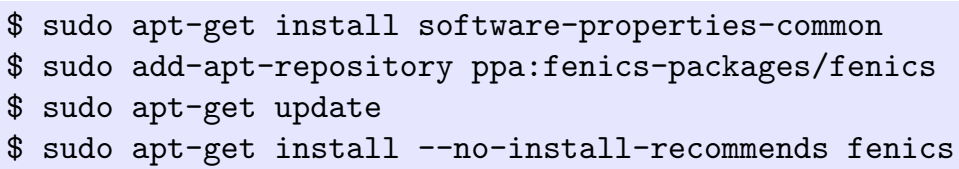

\footnotetext{
13 See https://github.com/SVMTK/SVMTK.

14 https://fenicsproject.org/download/
} 


\subsubsection{ParaView and other visualization tools}

We recommend using ParaView [6] to visualize the simulation results and other meshing objects that we discuss throughout the book. For installation instructions, see the ParaView website ${ }^{15}$. On Ubuntu Linux, ParaView can be installed with the following terminal command:

\$ sudo apt-get install paraview

(Ubuntu)

After installation, ParaView can be launched with

$\$$ paraview \&

An optional but useful tool for visualizing surfaces (in the form of surface STL files) is Gmsh [23]. For installation instructions, see the Gmsh website. ${ }^{16}$ On Ubuntu Linux, Gmsh can be installed with the following terminal command:

\$ sudo apt-get install gmsh

(Ubuntu)

For quick plotting in Python, the Python package Matplotlib is very convenient [32]. The pyplot module of Matplotlib can be imported in a Python script (as any other Python module) as:

import matplotlib.pyplot as plt

\subsubsection{Meshio for data and mesh conversion}

We recommend using meshio [56] for conversion between computational mesh formats. The meshio suite can convert between various unstructured mesh formats, for instance, between the CGAL medit file format (.mesh) and the FEniCS mesh format (.xml or .h5). We suggest using the pip installer to install meshio $^{17}$ for example, via typing the following in the terminal:

\footnotetext{
15 See https://www.paraview.org/.

16 See http://gmsh.info/\#Download.

17 See https://pypi.org/project/meshio/.
} 


\subsubsection{Testing the software pipeline}

To verify that all software dependencies are correctly installed, we provide a test script (in mri2fem/chp2); the test script can be executed with the command:

\section{\$ python3 test_book_software.py}

The script will check each software dependency and output a response indicating whether it is installed or not. If the software is not installed, the response provides a detailed description of ways to correctly install it, including links to installation guides:

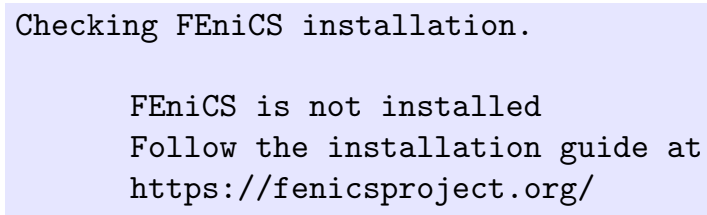

If the software is installed, the response is a single-line response confirming that the software is installed:

Checking FEniCS installation. => FEniCS installed

Open Access This chapter is licensed under the terms of the Creative Commons Attribution 4.0 International License (http://creativecommons.org/licenses/by/4.0/), which permits use, sharing, adaptation, distribution and reproduction in any medium or format, as long as you give appropriate credit to the original author(s) and the source, provide a link to the Creative Commons license and indicate if changes were made.

The images or other third party material in this chapter are included in the chapter's Creative Commons license, unless indicated otherwise in a credit line to the material. If material is not included in the chapter's Creative Commons license and your intended use is not permitted by statutory regulation or exceeds the permitted use, you will need to obtain permission directly from the copyright holder.

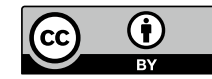

